\title{
Tumor-Suppressive microRNAs in Lung Cancer: Diagnostic and Therapeutic Opportunities
}

\author{
Lorenzo F. Sempere ${ }^{1}, \mathrm{Xi} \mathrm{Liu}^{2}$, and Ethan Dmitrovsky ${ }^{1,2,3, *}$ \\ Department of ${ }^{1}$ Medicine, ${ }^{2}$ Pharmacology and Toxicology, and ${ }^{3}$ Norris Cotton \\ Cancer Center, Dartmouth-Hitchcock Medical Center, Lebanon, NH and Dartmouth \\ Medical School, Hanover, NH \\ E-mail: Lorenzo.F.Sempere@Dartmouth.EDU; Xi.Liu@Dartmouth.EDU; Ethan.Dmitrovsky@Dartmouth.EDU
}

Received April 13, 2009; Accepted June 29, 2009; Published July 13, 2009

KEYWORDS: microRNA (miRNA, miR), lung cancer, expression profiling, in situ hybridization, miR-34c, miR-142-5p, miR145, cyclin E

Lung cancer is the leading cause of cancer-related death for both men and women in the U.S. and other countries. In 2008, 215,020 new cases were diagnosed and a total of 161,840 patients died from lung cancer in the U.S.[1]. Despite a substantial decrease in active smokers, which would eventually result in a considerable reduction in its incidence, lung cancer will continue to be a major worldwide health-care problem for several decades. This will be due both to current smokers and the large number of former smokers who are at high risk for developing lung cancer. Lung cancer, as for many other cancer types, is not a single disease, but rather a collection of diseases that present different histopathological, molecular, and clinical characteristics. Improved diagnostic and therapeutic tools are needed to better manage and treat those patients afflicted with lung cancer.

In a recent study by Liu and colleagues published in Clinical Cancer Research, a subset of microRNAs were pinpointed as candidate tumor-suppressive species in lung cancer[2]. Using new mouse transgenic models for lung cancer, genome-wide microRNA expression analyses identified a signature of down-regulated microRNAs in lung cancers relative to adjacent normal lung tissues. This signature was validated in clinical specimens from lung cancer patients, underscoring the relevance of these microRNAs to this human malignancy. The authors hypothesized that functions of these down-regulated microRNAs were detrimental to normal cells and their loss in tumors was an active process contributing to uncontrolled growth of the affected cells. Consistent with this hypothesis, in vitro experiments demonstrated that individually restoring miR-34c, miR-145, or miR-142-5p expression markedly reduced proliferation of murine lung cancer cell lines.

The microRNAs are a recently discovered class of short, noncoding, regulatory RNAs that constitute a new and broad layer of gene regulation[3]. Binding of the $~ 18-25$ nucleotide-long mature microRNAs to the 3'-untranslated region (UTR) of messenger RNAs (mRNAs) triggers translational down-regulation and/or increased degradation of the target gene products[3]. microRNA-mediated regulation of tumorigenesis has rapidly emerged as a promising area for translational cancer research. Altered expression of microRNAs has been linked to several cancer types, including lung cancer[4]. Moreover, altered tumor-suppressive and tumor-promoting functions of microRNAs have been shown to impact the course of tumorigenic processes greatly[5]. Thus, the potential clinical application of microRNAs as 
biomarkers and "druggable" targets is being intensely investigated in academic laboratories and pharmaceutical companies.

Liu and colleagues made use of novel mouse transgenic models for lung cancer driven by pulmonary epithelial-restricted expression of human wild-type or proteasome-resistant cyclin E; these mice developed lung adenocarcinomas[6]. Total RNA was independently extracted from adjacent normal and malignant lung tissues from these cyclin E-driven mouse models and hybridized against a locked nucleic acid (LNA) captured-probe microRNA microarray platform[7]. Differential expression of highlighted microRNAs was confirmed by quantitative real-time RT-PCR (qPCR) assays independently performed on RNA isolated from murine normal and malignant lung tissues. Notably, similar changes were observed in clinical specimens of different types of non-small cell lung cancers (NSCLC). These and other findings linked novel microRNAs to development of lung carcinogenesis.

To gain further insight into the spatial distribution of these highlighted microRNAs, in situ hybridization (ISH) assays based on LNA-modified probes were performed on formalin-fixed, paraffinembedded sections[8]. miR-34c and miR-145 were detected in different cell types in normal murine lung and were repressed in lung adenocarcinomas. miR-34c accumulated in bronchial epithelial cells, whereas miR-145 accumulated in smooth muscle cells of lamina muscularis and blood vessels. Intriguingly, miR$34 \mathrm{c}$ expression, as determined by qPCR assays, was substantially down-regulated in human large cell carcinoma, squamous cell carcinomas, and adenocarcinomas, but not in bronchioalveolar carcinomas (BACs). BACs generally have a slower progression and a more favorable clinical prognosis than other types of NSCLC. Although retention of miR-34c expression in these BACs might simply reflect the cell type of origin of these lesions, it is tempting to speculate that miR-34c activity enables the functioning of tumor-suppressive pathways such as p53 (see below). In this context, detection of miR-34c expression by qPCR and/or ISH assays could be used as a clinically relevant prognostic marker.

The miR-34 family members (miR-34a, miR34b, and miR-34c) are transcriptionally regulated by $\mathrm{p} 53$ and act in concert to relay p53 tumor-suppressive signals by negatively inhibiting cell cycle progression via binding to mRNA of target genes: cyclin D1 and/or its kinase binding partners CDK4/6[9]. Liu and colleagues unraveled cyclin $\mathrm{E}$ as an additional target of miR-34c to halt cell proliferation. Notably, cyclin $\mathrm{E}$ is the very oncogene genetically engineered to drive tumorigenesis in these mouse models for lung cancer. Similarly, let-7 was demonstrated to be a potent negative regulator of K-ras in a K-ras-driven mouse model for lung cancer[10]. This reciprocal negative regulation between an oncogenic driver and tumor-suppressive microRNA might be disproportionately represented in these models. Nonetheless, this highlights the ability and/or requirement of neoplastic cells to disrupt microRNA-mediated, tumorsuppressive pathways as a key step to become and/or remain malignant. Given the etiological relevance of cyclin $\mathrm{E}$ alterations in lung cancer, functional studies should be extended to human cancer cell lines and to an even larger panel of clinical specimens in order to validate this functional interaction between miR$34 \mathrm{c}$ and cyclin E. In a separate study, miR-145 was shown to be under the transcriptional control of p53 and to act to repress expression of oncogenic c-myc in breast and colon cancer cell lines[11]. Thus, it will be important to identify the target genes of miR-145 and miR-142-5p to further understand their role in lung cancer.

Synthetic compounds that mimic or antagonize microRNA activities have been developed and shown to be efficacious in restoring or depleting miRNA function in in vitro cell systems. Moreover, promising results have been reported in mouse and nonhuman primates, where cholesterol levels can be regulated by intravenous injection of anti-miR-122, nucleotide-based compounds[12]. Although in vivo delivery of synthetic compounds that restore microRNA activities is a challenging task, there is reason to be optimistic that this will be accomplished. Restoration of tumor-suppressive microRNAs, such as miR-34c and miR-145, perhaps in combination with depletion of oncogenic microRNAs, would represent intriguing strategies to combat lung cancer. 


\section{ACKNOWLEDGMENTS}

This work was supported by National Institutes of Health (NIH) and National Cancer Institute (NCI) grants RO1-CA087546, (E.D.), RO1-CA111422 (E.D.), RO3-CA130102 (E.D.), a Samuel Waxman Cancer Research Foundation Award (E.D.), the American Lung Association (X.L.), a postdoctoral fellowship (PDF0503563) grant from the Susan G. Komen Breast Cancer Foundation (L.S.), a Hitchcock Foundation grant (L.S.), and an AACR-Pancreatic Cancer Action Network Award (L.S). Ethan Dmitrovsky is an American Cancer Society Clinical Research Professor supported by a generous gift from F.M. Kirby Foundation.

\section{REFERENCES}

1. http://www.cancer.gov/cancertopics/types/lung

2. Liu, X., Sempere, L.F., Galimberti, F., Freemantle, S.J., Black, C., Dragnev, K.H., Ma, Y., Fiering, S., Memoli, V., Li, H., DiRenzo, J., Korc, M., Cole, C.N., Bak, M., Kauppinen, S., and Dmitrovsky, E. (2009) Uncovering growthsuppressive microRNAs in lung cancer. Clin. Cancer Res. 15, 1177-1183.

3. Carthew, R.W. and Sontheimer, E.J. (2009) Origins and mechanisms of miRNAs and siRNAs. Cell 136, $642-655$.

4. Barbarotto, E., Schmittgen, T.D., and Calin, G.A. (2008) MicroRNAs and cancer: profile, profile, profile. Int. J. Cancer 122, 969-977.

5. Ventura, A. and Jacks, T. (2009) MicroRNAs and cancer: short RNAs go a long way. Cell 136, 586-591.

6. Ma, Y., Fiering, S., Black, C., Liu, X., Yuan, Z., Memoli, V.A., Robbins, D.J., Bentley, H.A., Tsongalis, G.J., Demidenko, E., Freemantle, S.J., and Dmitrovsky, E. (2007) Transgenic cyclin E triggers dysplasia and multiple pulmonary adenocarcinomas. Proc. Natl. Acad. Sci. U. S. A. 104, 4089-4094.

7. Kauppinen, S., Vester, B., and Wengel, J. (2006) Locked nucleic acid: high-affinity targeting of complementary RNA for RNomics. Handb. Exp. Pharmacol. (173), 405-422.

8. Sempere, L.F., Christensen, M., Silahtaroglu, A., Bak, M., Heath, C.V., Schwartz, G., Wells, W., Kauppinen, S., and Cole, C.N. (2007) Altered microRNA expression confined to specific epithelial cell subpopulations in breast cancer. Cancer Res. 67, 11612-11620.

9. He, L., He, X., Lowe, S.W., and Hannon, G.J. (2007) microRNAs join the p53 network--another piece in the tumoursuppression puzzle. Nat. Rev. Cancer 7, 819-822.

10. Kumar, M.S., Erkeland, S.J., Pester, R.E., Chen, C.Y., Ebert, M.S., Sharp, P.A., and Jacks, T. (2008) Suppression of non-small cell lung tumor development by the let-7 microRNA family. Proc. Natl. Acad. Sci. U. S. A. 105, 39033908.

11. Sachdeva, M., Zhu, S., Wu, F., Wu, H., Walia, V., Kumar, S., Elble, R., Watabe, K., and Mo, Y.Y. (2009) p53 represses c-Myc through induction of the tumor suppressor miR-145. Proc. Natl. Acad. Sci. U. S. A. 106, 3207-3212.

12. Petri, A., Lindow, M., and Kauppinen, S. (2009) MicroRNA silencing in primates: towards development of novel therapeutics. Cancer Res. 69, 393-395.

\section{This article should be cited as follows:}

Sempere, L.F., Liu, X., and Dmitrovsky, E. (2009) Tumor-suppressive microRNAs in lung cancer: diagnostic and therapeutic opportunities. TheScientificWorldJOURNAL 9, 626-628. DOI 10.1100/tsw.2009.88. 

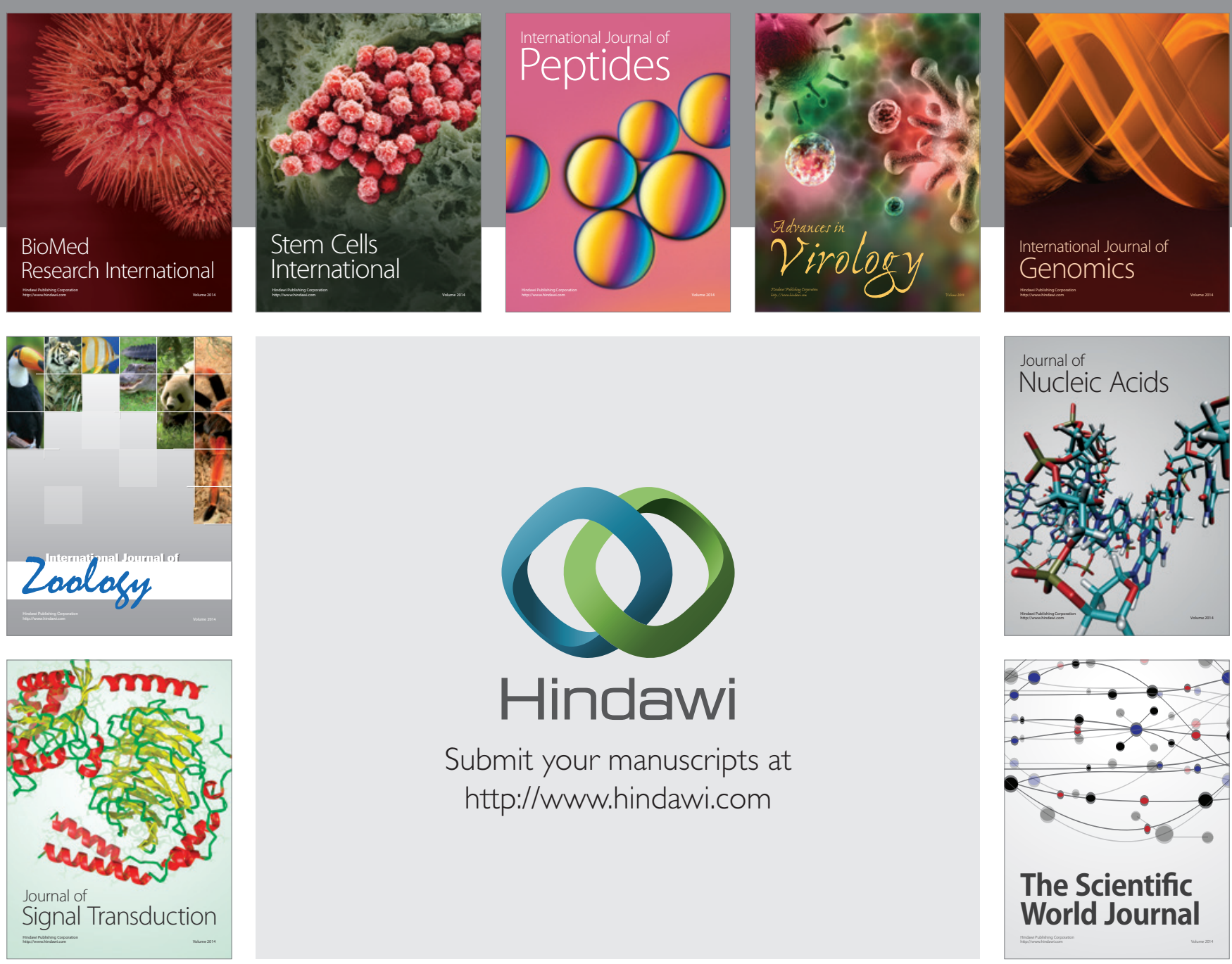

Submit your manuscripts at

http://www.hindawi.com
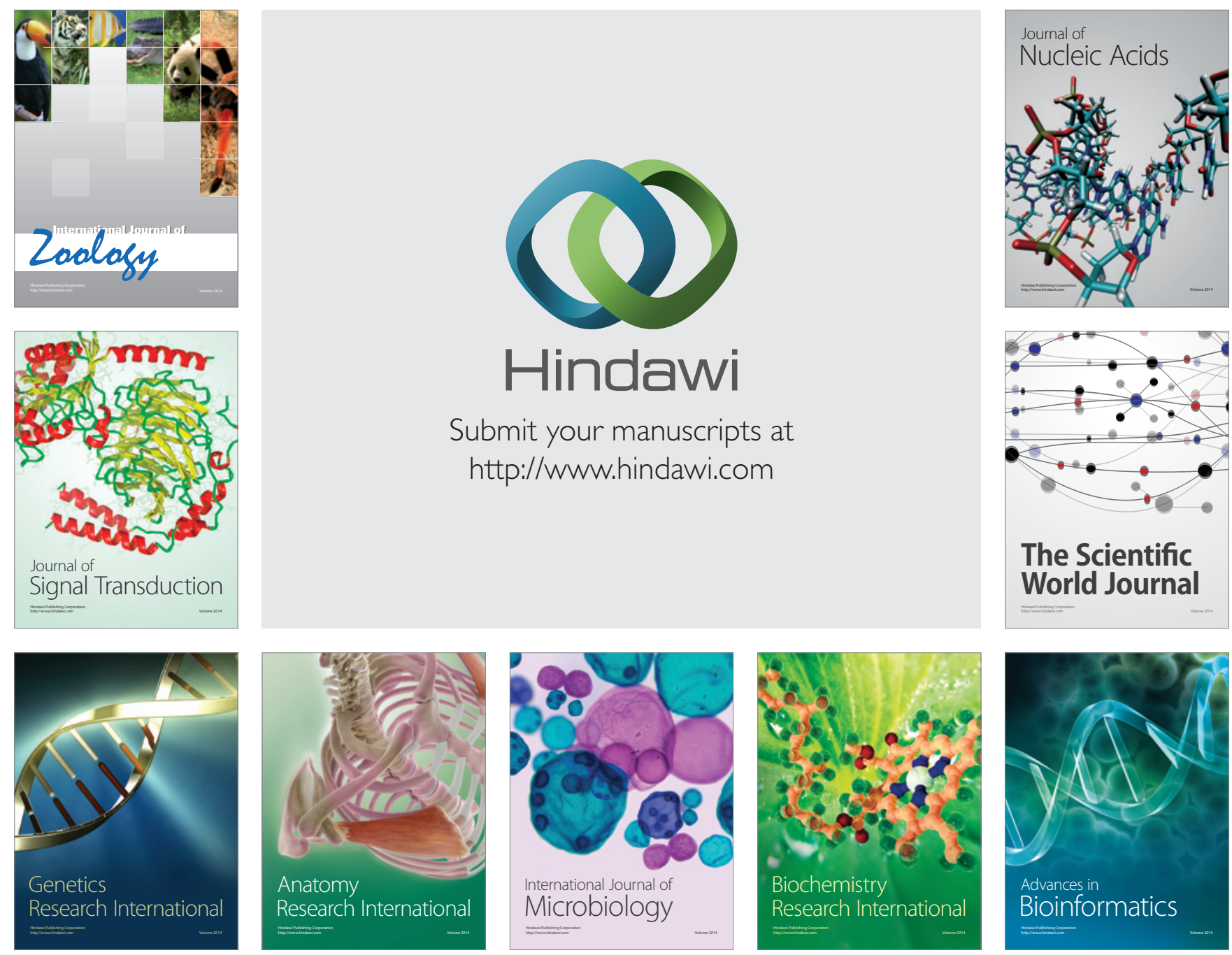

The Scientific World Journal
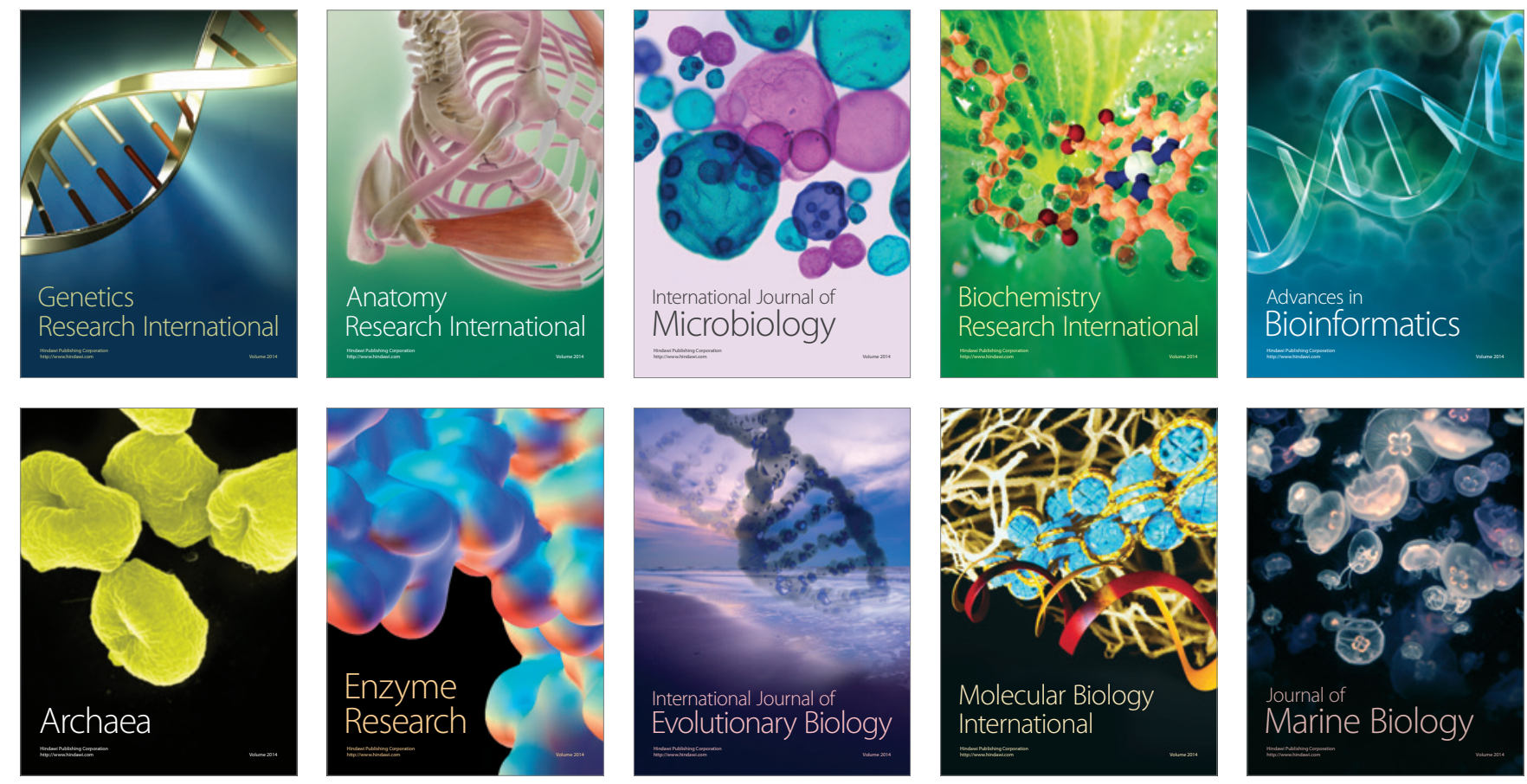